05.1;08.3

\title{
Упругое взаимодействие квантовых дисков в гибридных QD/NW-структурах
}

\author{
(C) А.Е. Романов ${ }^{1}$, А.Л. Колесникова ${ }^{1,2}$, М.Ю. Гуткин ${ }^{1-3}$, В.Е. Бугров ${ }^{1}$ \\ ${ }^{1}$ Университет ИТМО, Санкт-Петербург, Россия \\ ${ }^{2}$ Институт проблем машиноведения РАН, Санкт-Петербург, Россия \\ ${ }^{3}$ Санкт-Петербургский политехнический университет Петра Великого, Санкт-Петербург, Россия \\ E-mail: alexey.romanov@niuitmo.ru
}

Поступило в Редакцию 6 сентября 2021 г.

В окончательной редакции 24 сентября 2021 r.

Принято к публикации 24 сентября 2021 г.

Рассмотрено упругое взаимодействие квантовых дисков в нитевидном нанокристалле, т. е. в гибридной структуре квантовый диск/нитевидный нанокристалл с резкими гетерограницами. В рамках подхода микромеханики дефектов найдена энергия парного взаимодействия квантовых дисков и показано, что для квантовых дисков с решеточным несоответствием одного знака возникает зона притяжения друг к другу, зависящая от отношения аксиального размера квантового диска к радиусу нитевидного нанокристалла. Обнаруженный эффект следует учитывать при выборе режимов формирования гибридных структур квантовый диск/нитевидный нанокристалл и в моделях, объясняющих их свойства.

Ключевые слова: нитевидный нанокристалл, квантовый диск, QD/NW-гибридная структура, решеточное несоответствие, дилатационное включение, упругая энергия.

DOI: 10.21883/PJTF.2022.01.51878.19015

Гибридные полупроводниковые структуры, имеющие в своем составе как нитевидные нанокристаллы (nanowires) $(\mathrm{HHK}=\mathrm{NW})$, так и квантовые точки (quantum dots) (KT=QDT) или квантовые диски (quantum disks) (КД=QD), в настоящее время привлекают внимание широкого круга исследователей (см., например, обзоры $[1,2])$. Это связано с тем, что такие объекты обладают высоким структурным совершенством, что позволяет в полной мере реализоваться квантовым эффектам, присущим КТ, КД и ННК, без нежелательного дополнительного влияния дефектов кристаллической решетки, возникающих в планарных полупроводниковых гетероструктурах.

КТ и КД могут быть инкорпорированы в гибридные структуры в ННК различным образом [3]: располагаться на поверхности ННК [4], быть полностью погруженными в материал ННК [5-7] или занимать все сечение ННК $[8-10]$. В последнем случае, который и рассматривается в настоящей работе, речь идет об аксиальных QD/NW-гетероструктурах, имеющих большие перспективы практического применения в электронике [1] и фотонике [2].

КТ и КД представляют собой области ННК с измененными химическим составом и структурой и соответственно с отличными от остального объема материала физическими свойствами. Для дальнейшего анализа существенно наличие решеточного несоответствия

$$
\varepsilon_{m}=\frac{a_{\mathrm{QD}}-a_{\mathrm{NW}}}{a_{\mathrm{NW}}},
$$

где $a_{\mathrm{QD}}$ и $a_{\mathrm{NW}}-$ параметры кристаллических решеток в объеме КД (или КТ) и в объеме ННК соответственно.
Для простоты ограничимся дилатационным (равноосным) несоответствием, характерным для материалов c кубической элементарной ячейкой, что, например, имеет место для полупроводников $\mathrm{A}_{3} \mathrm{~B}_{5}$ со структурой сфалерита или для $\mathrm{Si}$ и $\mathrm{Ge}$ со структурой алмаза.

Хорошо известно, что при эпитаксиальном росте гетероструктур решеточное несоответствие обусловливает появление в материале упругих деформаций и механических напряжений, которые в свою очередь изменяют свойства полупроводников, а также приводят в ходе релаксации напряжений к появлению дислокационных дефектов [11]. В гибридных QD/NW-гетероструктурах в силу геометрических ограничений образование дислокаций затруднено, однако упругая энергия, вызванная решеточным несоответствием, должна приниматься во внимание при рассмотрении поведения подобных объектов. Именно поэтому настоящая работа посвящена анализу парного упругого взаимодействия КД в ННК.

В рамках подхода механики сплошной среды несоответствие $\varepsilon_{m}$ приобретает смысл собственной деформации (eigenstrain) [12], а КД оказывается упругим цилиндрическим включением конечной длины. Ранее был рассмотрен ряд задач по определению характеристик (напряжений и энергий) таких включений в бесконечном цилиндре, который служит адекватной моделью ННК. Обзор полученных результатов со ссылками на первоисточники дается в нашей недавней работе [13], где отмечено, что рассматривались включения с резким и плавным изменением собственной деформации вдоль оси цилиндра для случаев упругой изотропии и трансверсальной изотропии материала. Кроме того, исследовались упругие характеристики отдельных границ 
(переходных областей), разделяющих части цилиндра с постоянной собственной деформацией [13-15]. Контроль химического состава и напряженного состояния таких переходных областей важен для ННК с аксиальными гетероструктурами $[14,16]$.

На рис. 1 показана схема двух взаимодействующих дилатационных включений DI1 и DI2 в упругоизотропном цилиндре радиуса $a$. Размеры включений (толщина КД) в аксиальном направлении равны $h_{1}$ и $h_{2}$, а расстояние между ними - $L$. Компоненты собственных деформаций $\varepsilon_{i j}^{*}$ для каждого из включений определяются несоответствием $\varepsilon_{m}^{(1)}$ или $\varepsilon_{m}^{(2)}$. Например, для DI1

$$
\begin{aligned}
& \varepsilon_{r r}^{*(1)}(z)=\varepsilon_{\varphi \varphi}^{*(1)}(z)=\varepsilon_{z z}^{*(1)}(z) \\
& =\varepsilon_{m}^{(1)} H\left[\tilde{h}_{1} / 2-|\tilde{z}|\right] H[1-\tilde{r}],
\end{aligned}
$$

где использована цилиндрическая система координат $(r, \varphi, z) ; \tilde{r}=r / a, \tilde{z}=z / a, \tilde{h}_{1,2}=h_{1,2} / a, H[p]-$ ступенчатая функция Хевисайда.

Граничная задача теории упругости для изолированного дилатационного включения в упругоизотропном цилиндре была рассмотрена в работах $[13,15]$. В частности, для следа тензора напряжений $\sigma_{i i}^{(1)}$ было получено [13]:

$$
\begin{aligned}
& \sigma_{i i}^{(1)}=-\frac{4 G(1+v) \varepsilon_{m}^{(1)}}{1-v} H\left[\tilde{h}_{1} / 2-|\tilde{z}|\right] H[1-\tilde{r}] \\
& +\frac{8 G(1+v)^{2} \varepsilon_{m}^{(1)}}{\pi(1-v)} \int_{0}^{\infty} \frac{I_{1}(\beta) I_{0}(\tilde{r} \beta)}{\beta^{2} I_{0}^{2}(\beta)-\left(\beta^{2}-2 v+2\right) I_{1}^{2}(\beta)} \\
& \times \sin \frac{\tilde{h}_{1} \beta}{2} \cos \beta \tilde{z} d \beta
\end{aligned}
$$

где $G$ и $v$ - модуль сдвига и коэффициент Пуассона материала цилиндра; $I_{0}(\xi)$ и $I_{1}(\xi)$ - модифицированные функции Бесселя первого рода. В выражении (3) первый член в правой части задает след тензора (утроенную гидростатическую компоненту) напряжений дилатационного включения, находящегося в бесконечной среде, который равен нулю вне включения. Второй член в правой части в общем случае не равен нулю и возникает из-за ограничений, накладываемых на напряжения граничными условиями на свободной поверхности цилиндра. Именно его вклад определяет взаимодействие между дилатационными включениями в цилиндре.

В общем случае запасенная упругая энергия $E$, обусловленная произвольной собственной деформацией $\varepsilon_{i j}^{*}$, может найдена с использованием соотношения Муры [12]:

$$
E=-\frac{1}{2} \int_{V} \varepsilon_{i j}^{*} \sigma_{i j} d V
$$

где $\sigma_{i j}$ - напряжения, однозначно определяемые исследуемой собственной деформацией и граничными условиями, а интегрирование проводится по всему объему рассматриваемого упругого тела.

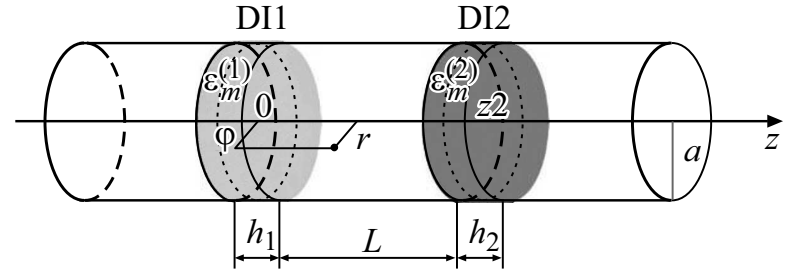

Рис. 1. Схема взаимодействующих квантовых дисков (дилатационных включений DI1 и DI2) в нитевидном нанокристалле - упругоизотропном цилиндре. Показаны параметры включений: решеточные несоответствия $\varepsilon_{m}^{(1)}$ и $\varepsilon_{m}^{(2)}$, аксиальные размеры $h_{1}$ и $h_{2}$ и координаты $z_{1}=0$ и $z_{2}$ в цилиндрической системе координат $(r, \varphi, z)$.

Для задачи нахождения упругой энергии системы взаимодействующих дилатационных включений выражение (4) сводится к следующему:

$$
E=-\frac{\varepsilon_{m}^{(1)}}{2} \int_{V^{(1)}} \sigma_{i i}^{(1)} d V-\frac{\varepsilon_{m}^{(2)}}{2} \int_{V^{(2)}} \sigma_{i i}^{(2)} d V-\varepsilon_{m}^{(2)} \int_{V^{(2)}} \sigma_{i i}^{(1)} d V
$$

где первые два члена в правой части - собственные энергии $E^{(1)}$ и $E^{(2)}$ изолированных (но находящихся в цилиндре!) дилатационных включений DI1 и DI2, а третий член - энергия парного взаимодействия $W(L)$, зависящая от расстояния $L$ между включениями в упругом цилиндре; $V^{(1,2)}=\pi a^{2} h_{1,2}$ - объемы включений.

Расчеты с использованием формул (2), (3) и (5) дают

$$
\begin{aligned}
& E^{(1,2)}=\frac{2 G(1+v)\left(\varepsilon_{m}^{(1,2)}\right)^{2}}{1-v} V^{(1,2)}-\frac{16 G(1+v)^{2}\left(\varepsilon_{m}^{(1,2)}\right)^{2} a^{3}}{1-v} \\
& \times \int_{0}^{\infty} \frac{I_{1}^{2}(\beta)}{\beta^{2} I_{0}^{2}(\beta)-\left(\beta^{2}-2 v+2\right) I_{1}^{2}(\beta)} \frac{1}{\beta^{2}}\left(\sin \frac{\tilde{h}_{1,2} \beta}{2}\right)^{2} d \beta \\
& W(L)=-\frac{32 G(1+v)^{2} \varepsilon_{m}^{(1)} \varepsilon_{m}^{(2)} a^{3}}{1-v} \\
& \quad \times \int_{0}^{\infty} \frac{I_{1}^{2}(\beta)}{\beta^{2} I_{0}^{2}(\beta)-\left(\beta^{2}-2 v+2\right) I_{1}^{2}(\beta)} \frac{1}{\beta^{2}} \sin \frac{\tilde{h}_{1} \beta}{2} \sin \frac{\tilde{h}_{2} \beta}{2} \\
& \times \cos \left(\tilde{L}+\frac{\tilde{h}_{1}}{2}+\frac{\tilde{h}_{2}}{2}\right) \beta d \beta,
\end{aligned}
$$

где $\tilde{L}=L / a$. При $L=0$ включения примыкают друг к другу. Случай перекрытия включений не рассматривается.

Первый член в правой части формулы (6), которая впервые была получена в работе [15], задает пропорциональную объему энергию дилатационного включения в бесконечной среде, а второй показывает уменьшение энергии единичного включения за счет экранирования упругих полей свободной поверхностью цилиндра. Если рассмотреть два дилатационных включения в бесконечной упругой среде, то можно убедиться, что энергия 


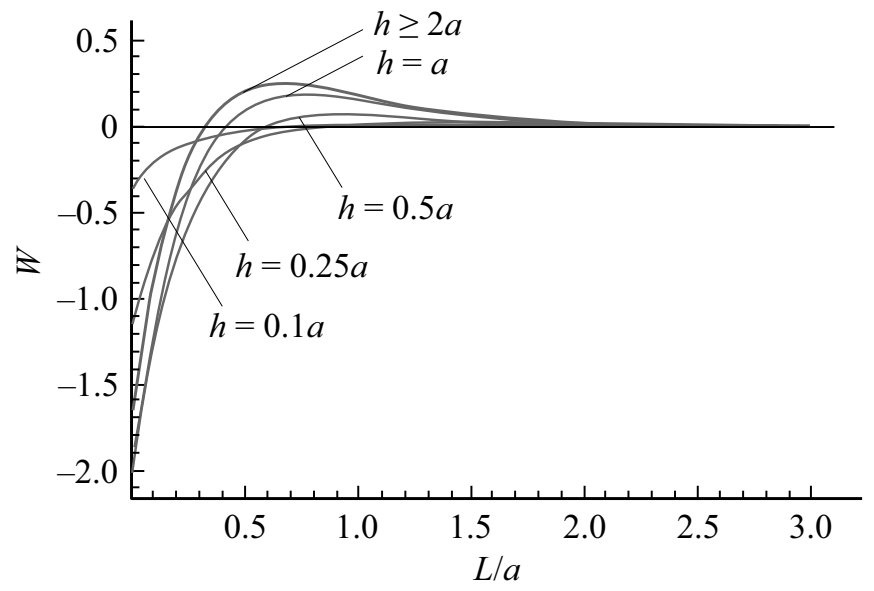

Рис. 2. Энергия взаимодействия двух одинаковых квантовых дисков (дилатационных включений) в зависимости от расстояния между ними вдоль оси нитевидного нанокристалла упругого цилиндра. Энергия дана в единицах $G \varepsilon_{m}^{2} a^{3}$, где $G$ - модуль сдвига, $\varepsilon_{m}$ - решеточное несоответствие КД относительно материала ННК, $a-$ радиус цилиндра. Расчеты сделаны для коэффициента Пуассона $v=0.3$.

их взаимодействия (при отсутствии перекрытия объемов включений) равна нулю, поскольку вне включения гидростатическая компонента напряжения отсутствует. В цилиндре, как следует из соотношения (3), такая компонента имеет место, что приводит к ненулевому взаимодействую дилатационных включений, задаваемому соотношением (7), а следовательно, к упругому взаимодействию КД в ННК.

Зависимость энергии взаимодействия $W$ от расстояния $L$ между равновеликими дилатационными КД $\left(h_{1}=h_{2}=h, \varepsilon_{m}^{(1)}=\varepsilon_{m}^{(2)}=\varepsilon_{m}\right)$ в цилиндре показана на рис. 2. Видно, что КД с произвольным аксиальным размером (проверено для $h \geqslant 0.05 a$, что при $a=50 \mathrm{~nm}$ составляет $2.5 \mathrm{~nm}$ ) демонстрируют отрицательную энергию взаимодействия при малых $L$, затем выход энергии из отрицательной зоны при увеличении $L$ и наличие максимума энергии $W_{\max }(h)$ в областях от $L \sim 0.65 a-0.66 a$ при $h \geqslant 2 a$ до $L \sim 1.3 a$ при $h \approx 0.1 a$. Энергия взаимодействия двух КД становится нечувствительной к параметру $h$ при $h \geqslant 2 a$. Кроме того, она практически равна нулю при разведении КД на расстояние $L \approx 2.5 a$.

Полученные результаты открывают путь к дальнейшему исследованию различных релаксационных процессов в ННК, приводящих к уменьшению их энергии деформации, например, за счет перераспределения и сегрегации примесей или за счет образования дислокаций несоответствия, что находится в центре наших текущих интересов. Обнаруженный в настоящей работе эффект взаимного притяжения КТ с трехмерной собственной дилатацией ставит вопрос о размерной устойчивости подобных гетероструктур. Отметим, что эффект притяжения (отталкивания) точечных дефектов с собственной дилатацией одного (противоположного) знака имеет место для их упругого взаимодействия вблизи плоской свободной поверхности [17], а также для дилатационных дефектов, находящихся в упругоанизотропном материале [18].

Таким образом, поставлена и решена задача об упругом взаимодействии квантовых дисков в гибридных QD/NW-структурах, в которых материал дисков обладает решеточным несоответствием по отношению к материалу нитевидного нанокристалла. Для квантовых дисков использовано представление в виде включений с дилатационной собственной деформацией, внедренных в виде вставок в материал нитевидного нанокристалла. Обнаружен неизвестный ранее эффект притяжения квантовых дисков с дилатацией одного знака и показано, что величина этого эффекта определяется приведенной (по отношению к радиусу ННК) толщиной взаимодействующих квантовых дисков.

\section{Финансирование работы}

Работа выполнена при поддержке Министерства науки и высшего образования РФ (проект тематики научных исследований № 2019-1442).

\section{Конфликт интересов}

Авторы заявляют, что у них нет конфликта интересов.

\section{Список литературы}

[1] Ch. Jia, Zh. Lin, Y. Huang, X. Duan, Chem. Rev., 119, 9074 (2019). DOI: 10.1021/acs.chemrev.9b00164

[2] L.N. Quan, J. Kang, C.-Zh. Ning, P. Yang, Chem. Rev., 119, 9153 (2019). DOI: 10.1021/acs.chemrev.9b00240

[3] M. de la Mata, X. Zhou, F. Furtmayr, J. Teubert, S. Gradecak, M. Eickhoff, A. Fontcuberta i Morral, J. Arbiol, J. Mater. Chem. C, 1, 4300 (2013). DOI: 10.1039/c3tc30556b

[4] E. Uccelli, J. Arbiol, J.R. Morante, A. Fontcuberta i Morral, ACS Nano, 4, 5985 (2010). DOI: 10.1021/nn101604k

[5] A. Tribu, G. Sallen, T. Aichele, R. Andre, J.-P. Poizat, C. Bougerol, S. Tatarenko, K. Kheng, Nano Lett., 8, 4326 (2008). DOI: $10.1021 / \mathrm{nl} 802160 \mathrm{z}$

[6] V.N. Kats, V.P. Kochereshko, A.V. Platonov, T.V. Chizhova, G.E. Cirlin, A.D. Bouravleuv, Yu.B. Samsonenko, I.P. Soshnikov, E.V. Ubyivovk, J. Bleuse, H. Mariette, Semicond. Sci. Technol., 27, 015009 (2012). DOI: 10.1088/0268-1242/27/1/015009

[7] L. Leandro, J. Hastrup, R. Reznik, G. Cirlin, N. Akopian, NPJ Quant. Inf., 6, 93 (2020). DOI: 10.1038/s41534-020-00323-9

[8] M. Tchernycheva, G.E. Cirlin, G. Patriarche, L. Travers, V. Zwiller, U. Perinetti, J.-Ch. Harmand, Nano Lett., 7, 1500 (2007). DOI: 10.1021/n10702281

[9] E. Sutter, P. Sutter, Chem. Mater., 31, 8174 (2019). DOI: $10.1021 /$ acs.chemmater.9b03000

[10] A.D. Bolshakov, V.V. Fedorov, N.V. Sibirev, M.V. Fetisova, E.I. Moiseev, N.V. Kryzhanovskaya, O.Yu. Koval, E.V. Ubyivovk, A.M. Mozharov, G.E. Cirlin, I.S. Mukhin, Phys. Status Solidi RRL, 13, 1900350 (2019). DOI: $10.1002 /$ pssr.201900350 
[11] L.B. Freund, S. Suresh, Thin film materials: stress, defect formation and surface evolution (Cambridge University Press, 2009).

[12] T. Mura, Micromechanics of defects in solids (Martinus Nijhoff, Boston, 1987).

[13] A.E. Romanov, A.L. Kolesnikova, M.Yu. Gutkin, Int. J. Solids Struct., 213, 121 (2021). DOI: 10.1016/j.ijsolstr.2020.12.010

[14] F. Glas, Phys. Rev. B, 74, 121302(R) (2006). DOI: $10.1103 /$ PhysRevB.74.121302

[15] A.E. Romanov, A.L. Kolesnikova, M.Yu. Gutkin, V.G. Dubrovskii, Scripta Mater., 176, 42 (2020). DOI: $10.1016 /$ j.scriptamat.2019.09.036

[16] D.V. Beznasyuk, P. Stepanov, J.L. Rouviere, F. Glas, M. Verheijen, J. Claudon, M. Hocevar, Phys. Rev. Mater., 4, 074607 (2020). DOI: 10.1103/PhysRevMaterials.4.074607

[17] R.D. Mindlin, in Proc. First Midwestern Conf. on solid mechanics (Urbana, Illinois, 1953), p. 56.

[18] С.А. Кукушкин, А.В. Осипов, Р.С. Телятник, ФТТ, 58, 941 (2016). [S.A. Kukushkin, A.V. Osipov, R.S. Telyatnik, Phys. Solid State, 58, 971 (2016).

DOI: $10.1134 / \mathrm{S} 1063783416050140]$. 